\title{
EVALUATION OF THE JET DAMPING EFFECT ON FLIGHT DYNAMICS OF A HOMING GUIDED MISSILE
}

\author{
Grzegorz KowALECZKo \\ Air Force Institute of Technology, Warsaw, Poland \\ e-mail: g.kowaleczko@chello.pl
}

The paper presents evaluation of the jet damping effect on spatial motion of a homing guided missile with variable mass. The mathematical model of motion including effects generated by the burning fuel are presented - changes of mass characteristics as well as the jet damping effect are taken into account. Both the influences of inertia forces/moments and changes of the position of mass center are calculated. The damping effect generating additional forces and moments acting on the missile is also determined. The obtained set of equations of motion allows one to analyze a wide spectrum of various problems, e.g. the influence of jet damping on the homing guided missile trajectory or the dynamic response of the missile to atmospheric disturbances. Exemplary results of simulations are shown.

Keywords: missile dynamics, variable mass system, jet damping effect, numerical simulations

\section{Introduction}

During the phase of engine working, a missile should be treated as a variable mass system (Davis et al., 1958; Dimitrevskii, 1972; Quarelli et al., 2014; Thomson, 1965a,b, 1986). The combustion process causes a change in the mass center - all mass characteristics of the missile change. Simultaneously produced gases reach high speed inside the engine. In the case of pitching or yawing motion of the missile, these moving gases are a source of the "jet damping" effect. This effect influences rotational motion of the missile. Usually, this effect is ignored because the burning phase is short and the blast-off parameters are stable. In particular, it concerns the angular velocities which are equal to zero. But in the case of a homing guided missile with a long burning process, it may be possible to observe its maneuvers when the engine still works and angular velocities are different from zero. It means that the jet damping effect occurs. It should be assessed whether it is important for the dynamics of missile flight and for precision of target hitting.

To calculate the jet damping effect one has to know the velocity distribution of combustion gases inside the engine. Because the engine consists of two parts - a combustion chamber and an exhaust nozzle, we have to determine these distributions inside both engine components. In the case of the combustion chamber, the combustion law and the geometry of the fuel rod have to be taken into account, and usually an analytical formula describing velocity can be determined. For the exhaust nozzle, thermodynamic laws for supersonic de Laval nozzle are included into consideration. In this case, the velocity profile can be determined numerically - an analytical formula is not possible because of various descriptions of nozzle geometry.

\section{Geometry and kinematics of missile motion}

\subsection{Geometry of the missile}

The following right-handed rectangular coordinate systems are applied in order to determine the set of equations of motion: 
1. $O_{I} x_{I} y_{I} z_{I}$ inertial coordinate system with the origin at any point in space;

2. $O_{Z} x_{I} y_{I} z_{I}$ - coordinate system with the origin at the center of the Earth parallel to $O_{I} x_{I} y_{I} z_{I}$

3. $O_{g} x_{g} y_{g} z_{g}$ - moving coordinate system with the origin $O_{g}$ at the Earth surface;

4. $O x_{g} y_{g} z_{g}$ - moving coordinate system parallel to $O_{g} x_{g} y_{g} z_{g}$ with the origin $O$ at any fixed point of the missile;

5. Oxyz - moving coordinate system with the origin at any fixed point of the missile.

\subsection{Transformations of coordinate systems}

If one has components of any vector in any coordinate system, its components in another system can be calculated using a transformation matrix. This matrix is determined using elementary angles of rotation. These angles define the relative position of two coordinate systems.

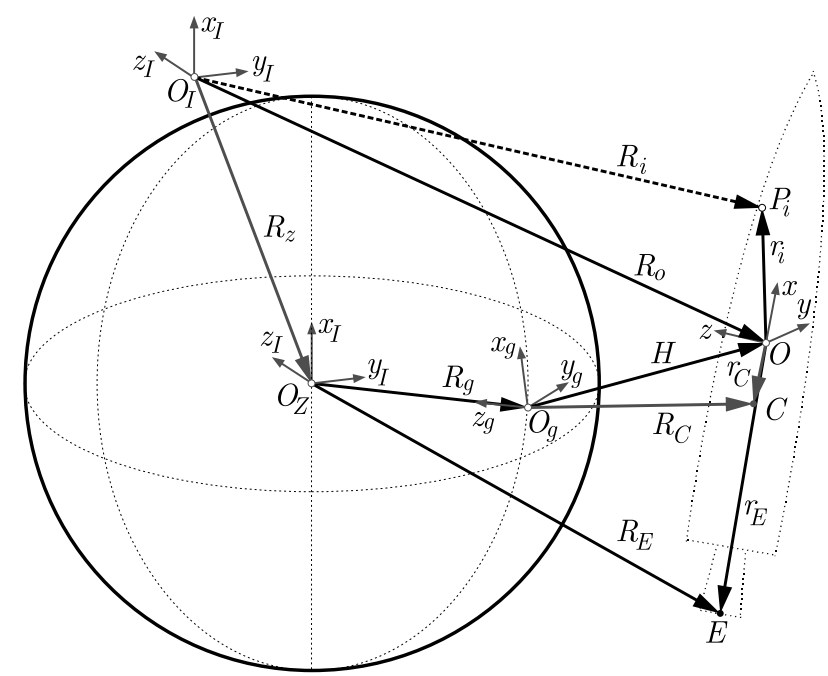

Fig. 1. Coordinate systems $O_{I} x_{I} y_{I} z_{I}, O x y z$ and basic vectors

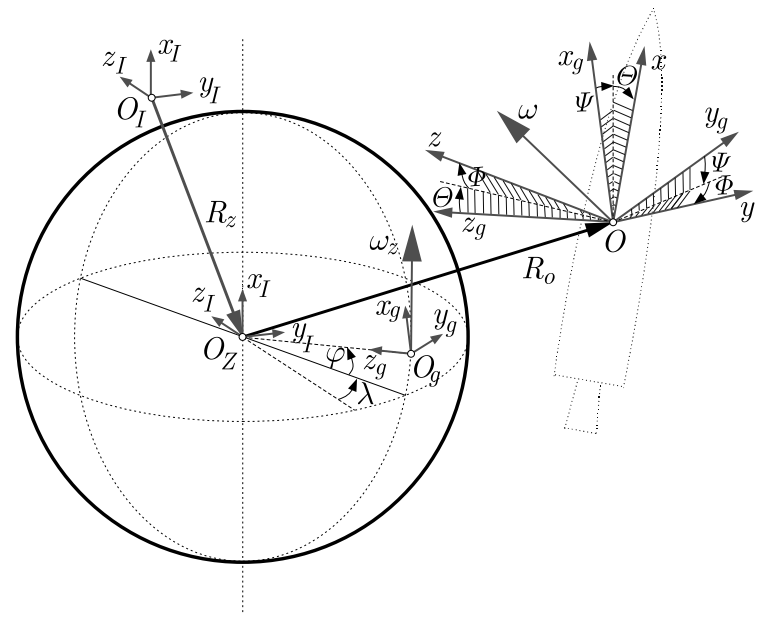

Fig. 2. Transformations of the coordinate systems

For the transformation from $O_{Z} x_{I} y_{I} z_{I}$ to $O_{g} x_{g} y_{g} z_{g}$, the following angles (see Fig. 2) are used: $\lambda$ - azymuth, $\varphi$ - declination. For the systems $O_{g} x_{g} y_{g} z_{g}$ and $O x y z$, the following angles are used: $\Psi$ - yaw, $\Theta$ - pitch, $\Phi$ - roll. The transformation matrices have the form, respectively 


$$
\begin{aligned}
\mathbf{L}_{g / I}= & {\left[\begin{array}{ccc}
\cos \phi & -\sin \phi \sin \lambda & \sin \phi \cos \lambda \\
0 & \cos \lambda & \sin \lambda \\
-\sin \phi & -\cos \phi \sin \lambda & \cos \phi \cos \lambda
\end{array}\right] } \\
\mathbf{L}_{m / g}= & {\left[\begin{array}{ccc}
\cos \Psi \cos \Theta & \sin \Psi \cos \Theta & -\sin \Theta \\
\cos \Psi \sin \Theta \sin \Phi-\sin \Psi \cos \Phi & \sin \Psi \sin \Theta \sin \Phi+\cos \Psi \cos \Phi & \cos \Theta \sin \Phi \\
\cos \Psi \sin \Theta \cos \Phi+\sin \Psi \sin \Phi & \sin \Psi \sin \Theta \cos \Phi-\cos \Psi \sin \Phi & \cos \Theta \cos \Phi
\end{array}\right] }
\end{aligned}
$$

\subsection{Velocities}

\subsubsection{Angular velocities}

We define the following angular velocities: $\omega_{z}$ - angular velocity of $O_{g} x_{g} y_{g} z_{g}$ relative to $O_{g} x_{I} y_{I} z_{I}$. This velocity in $O_{g} x_{I} y_{I} z_{I}$ has the following components: $\boldsymbol{\omega}_{z}=\left[\omega_{z}, 0,0\right]^{\mathrm{T}} ; \boldsymbol{\omega}$ - angular velocity of $O x y z$ relative to $O_{g} x_{g} y_{g} z_{g}$. This velocity in $O x y z$ has the following components: $\boldsymbol{\omega}=[P, Q, R]^{\mathrm{T}} ; \boldsymbol{\Omega}$ - angular velocity of $O x y z$ relative to the inertial coordinate system. It is equal to the sum of two velocities $\boldsymbol{\Omega}=\boldsymbol{\omega}+\boldsymbol{\omega}_{z}$.

\subsubsection{Linear velocities}

In further analysis, one assumes that the inertial system with the origin at the center of the Earth is the reference coordinate system (i.e. $\mathbf{R}_{Z}=\mathbf{0}$ ). The translational and rotational motion of the missile is represented by a change of the vector $\mathbf{R}_{O}$. The absolute velocity of any point of the missile $P_{i}$ is defined by the expression

$$
\mathbf{V}_{i}=\mathbf{V}_{O / g}+\mathbf{V}_{r e l_{\imath} i}+\boldsymbol{\omega} \times \mathbf{r}_{i}+\boldsymbol{\omega}_{z} \times \mathbf{R}_{i}
$$

where $\mathbf{V}_{O / g}=[U, V, W]^{\mathrm{T}}$ is the velocity of the pole $O, \mathbf{V}_{\text {rel_ } i}=d^{\prime} \mathbf{r}_{i} / d t$ is the relative velocity in the case if the point $P_{i}$ represents a gas particle, which after fuel burning moves inside the missile body in the direction to the nozzle - in this case, this motion causes a change of the vector $\mathbf{r}_{i}$. If the angular velocity of the Earth is omitted, the last component is equal to zero.

\subsection{Accelerations}

Newton's laws hold true in the inertial system. The absolute acceleration of the point $P_{i}$ in this system is equal to

$$
\begin{aligned}
\mathbf{a}_{i}= & \mathbf{a}_{O / g}+\boldsymbol{\omega} \times \mathbf{V}_{O / g}+\boldsymbol{\varepsilon} \times \mathbf{r}_{i}+\mathbf{a}_{r e l \_i}+\boldsymbol{\omega} \times\left(\boldsymbol{\omega} \times \mathbf{r}_{i}\right)+2\left(\boldsymbol{\omega}+\boldsymbol{\omega}_{z}\right) \times \mathbf{V}_{r e l_{\_} i} \\
& +2 \boldsymbol{\omega}_{z} \times \mathbf{V}_{O / g}+2\left(\boldsymbol{\omega}_{z} \times \boldsymbol{\omega}\right) \times \mathbf{r}_{i}+2 \boldsymbol{\omega} \times\left(\boldsymbol{\omega}_{z} \times \mathbf{r}_{i}\right)+\boldsymbol{\omega}_{z} \times\left(\boldsymbol{\omega}_{z} \times \mathbf{R}_{i}\right)
\end{aligned}
$$

where $\mathbf{a}_{O / g}=d^{\prime} \mathbf{V}_{O / g} / d t$ is the acceleration of the pole $O$ in $O_{g} x_{g} y_{g} z_{g} ; \boldsymbol{\varepsilon}=d^{\prime} \boldsymbol{\omega} / d t$ is the angular acceleration of the missile; $\mathbf{a}_{\text {rel_i } i}=d \mathbf{V}_{\text {rel_i }} / d t$ is the relative acceleration of a particle moving inside the missile. For missiles of short and medium-range, the issues related to the angular velocity of the Earth can be omitted. In this case, the absolute acceleration is equal to

$$
\mathbf{a}_{i}=\mathbf{a}_{O}+\boldsymbol{\omega} \times \mathbf{V}_{O}+\boldsymbol{\varepsilon} \times \mathbf{r}_{i}+\mathbf{a}_{r e l \_i}+\boldsymbol{\omega} \times\left(\boldsymbol{\omega} \times \mathbf{r}_{i}\right)+2 \boldsymbol{\omega} \times \mathbf{V}_{r e l \_i}
$$

In this case, $O_{g} x_{g} y_{g} z_{g}$ is the inertial frame. Accordingly, in the above formula, the subscript $O / g$ is replaced by $O$. 


\section{Mass and mass center of the missile}

The missile is a system of variable mass. Its mass is the sum of all elementary masses contained in a given time within its body. During the active phase of flight, this mass includes: fuselage, fuel, gases contained in the combustion chamber and in the nozzle: $m=m_{\text {fus }}+m_{\text {fuel }}+m_{\text {chamber }}+$ $m_{n o z z l e}$. The position of the center of mass in the inertial system is determined by the vector $\mathbf{R}_{C}=\left(\sum_{i} \mathbf{R}_{i} m_{i}\right) / m$. However, in the moving system $O x y z$, the position of the center of mass is determined by the formula $\mathbf{r}_{C}=\mathbf{S} / m$, where $\mathbf{S}$ is the vector of static moments

$$
\mathbf{S}=\sum_{i} \mathbf{r}_{i} m_{i}=\left[\sum_{i} x_{i} m_{i}, \sum_{i} y_{i} m_{i}, \sum_{i} z_{i} m_{i}\right]^{\mathrm{T}}
$$

\section{The equations of translatory motion of the missile with variable mass}

The momentum of the missile with variable mass and with one outlet nozzle is

$$
\mathbf{p}(t)=m \mathbf{V}_{C}+\dot{m}\left(\mathbf{R}_{C}-\mathbf{R}_{E}\right)
$$

where $\dot{m}=d m / d t$ is the mass flow rate; $\mathbf{V}_{C}=d \mathbf{R}_{C} / d t$ is the absolute velocity of the center of mass; $\mathbf{R}_{E}=\mathbf{R}_{O}+\mathbf{r}_{E}$ is the vector determining the position of the nozzle. On the basis of equation (4.1), one can calculate the total differential of momentum for the mass inside the body

$$
d \mathbf{p}_{1}(t)=\left[\dot{m} \mathbf{V}_{C}+m \frac{d \mathbf{V}_{C}}{d t}+\ddot{m}\left(\mathbf{R}_{C}-\mathbf{R}_{E}\right)+\dot{m}\left(\mathbf{V}_{C}-\mathbf{V}_{E}\right)\right] d t
$$

For the mass $\dot{m} d t$, which lefts the missile body with the absolute velocity $\mathbf{V}_{e}^{*}$, the total differential of momentum is equal to $d \mathbf{p}_{2}(t)=-\dot{m} d t \mathbf{V}_{e}^{* 1}$. Finally, the change of momentum of both the missile as well as the mass $\dot{m} d t$ is equal to

$$
d \mathbf{p}=d \mathbf{p}_{1}+d \mathbf{p}_{2}=\left[m \frac{d \mathbf{V}_{C}}{d t}+\ddot{m}\left(\mathbf{R}_{C}-\mathbf{R}_{E}\right)+\dot{m}\left(2 \mathbf{V}_{C}-\mathbf{V}_{E}-\mathbf{V}_{e}^{*}\right)\right] d t
$$

According to the second Newton law, the change of momentum of the missile is equal to the impulse of the external force $\mathbf{F}$

$$
d \mathbf{p}(t)=\mathbf{F} d t
$$

On the basis of expressions (4.3) and (4.4) one can obtain

$$
m \frac{d \mathbf{V}_{C}}{d t}+\ddot{m}\left(\mathbf{R}_{C}-\mathbf{R}_{E}\right)+\dot{m}\left(2 \mathbf{V}_{C}-\mathbf{V}_{E}-\mathbf{V}_{e}^{*}\right)=\mathbf{F}
$$

Without taking into account the angular velocity of the Earth rotation, the absolute velocities of points $C$ and $E$ are defined by expressions

$$
\mathbf{V}_{C}=\mathbf{V}_{O}+\frac{d^{\prime} \mathbf{r}_{C}}{d t}+\boldsymbol{\omega} \times \mathbf{r}_{C} \quad \mathbf{V}_{E}=\mathbf{V}_{O}+\boldsymbol{\omega} \times \mathbf{r}_{E}
$$

The second component of $\mathbf{V}_{C}$ is a relative velocity of the center of mass due to its motion as a result of the burning process $\mathbf{V}_{\text {rel_C }}=d^{\prime} \mathbf{r}_{C} / d t$. It does not occur in $\mathbf{V}_{E}$ because the position of the exit nozzle relative to the pole $O$ is fixed. The absolute velocity of the gases leaving the

\footnotetext{
${ }^{1}$ The sign "-" shows that the mass of the missile decreases and the value of the mass flow rate is negative.
} 
outlet nozzle $\mathbf{V}_{e}^{*}$ is equal to the sum of the nozzle exit velocity $\mathbf{V}_{E}$ and the relative velocity $\mathbf{U}_{r e l}$, which is a result of the thermodynamic processes inside the nozzle

$$
\mathbf{V}_{e}^{*}=\mathbf{V}_{E}+\mathbf{U}_{r e l}=\mathbf{V}_{O}+\boldsymbol{\omega} \times \mathbf{r}_{E}+\mathbf{U}_{r e l}
$$

Taking into account the above terms in equation (4.5), we obtain

$$
m \frac{d \mathbf{V}_{C}}{d t}+\ddot{m}\left(\mathbf{r}_{C}-\mathbf{r}_{E}\right)+2 \dot{m}\left[\mathbf{V}_{r e l} C+\boldsymbol{\omega} \times\left(\mathbf{r}_{C}-\mathbf{r}_{E}\right)\right]=\mathbf{F}+\mathbf{T}
$$

where the thrust of the engine is $\mathbf{T}=\dot{m} \mathbf{U}_{r e l}$. The thrust $\mathbf{T}$ is positive because in the system Oxyz both the value of the mass flow rate $\dot{m}$ as well as the relative velocity $\mathbf{U}_{r e l}$ are negative.

Taking into account formula (2.4) related to the center of mass, on the basis of (4.8), one can determine the equation of translatory motion of the missile with variable mass

$$
\begin{aligned}
& m\left(\frac{d^{\prime} \mathbf{V}_{O}}{d t}+\boldsymbol{\omega} \times \mathbf{V}_{O}\right)+\varepsilon \times \mathbf{S}+\boldsymbol{\omega} \times(\boldsymbol{\omega} \times \mathbf{S})+m \mathbf{a}_{r e l_{-} C}+2 m \boldsymbol{\omega} \times \mathbf{V}_{r e l_{-} C} \\
& \quad+\ddot{m}\left(\mathbf{r}_{C}-\mathbf{r}_{E}\right)+2 \dot{m}\left[\mathbf{V}_{r e l_{-} C}+\boldsymbol{\omega} \times\left(\mathbf{r}_{C}-\mathbf{r}_{E}\right)\right]=\mathbf{F}+\mathbf{T}
\end{aligned}
$$

If the thrust has only one component $T_{x}=T$, the missile is axisymmetric and the center of mass and the center of the missile nozzle exit are all the time on the axis of the missile, then we have three scalar equations

$$
\begin{aligned}
& m(\dot{U}+Q W-R V)-S_{x}\left(Q^{2}+R^{2}\right)+m a_{x \_r e l_{-} C}+\ddot{m}\left(x_{C}-x_{E}\right)+2 \dot{m} U_{r e l_{-} C}=F_{x}+T \\
& m(\dot{V}+R U-P W)+S_{x}(P Q+\dot{R})+2 m R U_{r e l_{-} C}+2 \dot{m} R\left(x_{C}-x_{E}\right)=F_{y} \\
& m(\dot{W}+P V-Q U)+S_{x}(P R-\dot{Q})-2 m Q U_{r e l_{-} C}-2 \dot{m} Q\left(x_{C}-x_{E}\right)=F_{z}
\end{aligned}
$$

\section{The equations of rotational motion of the missile with variable mass}

For rotational motion the second law of dynamics relates to the derivative (with respect to time) of the angular momentum of the system of material points. Consideration should be taken for the same mass particles at two successive moments of time.

- At the time $t$, the system consists of mass $m=\sum_{i} m_{i}$. Locations of elementary masses mi are determined by vectors $\mathbf{R}_{i}$ (in the inertial system) or by vectors $\mathbf{r}_{i}$ (in the moving system). The absolute velocity is $\mathbf{V}_{i}$. The angular momentum $\mathbf{K}_{O}$ determined in relation to the pole $O$ (Fig. 1) is equal to

$$
\mathbf{K}_{O}(t)=\sum_{i} \mathbf{K}_{O i}=\sum_{i} \mathbf{r}_{i} \times \mathbf{p}_{i}=\sum_{i} \mathbf{r}_{i} \times m_{i} \mathbf{V}_{i}
$$

- At the time $t+\Delta t$, the system consists of two parts:

- mass $m=\sum_{i}\left(m_{i}-\Delta m_{i}\right)$ that does not leave the body of the missile. The change of mass is equal to $\Delta m_{i}=-\dot{m}_{i} \Delta t$. Locations of the elementary masses are determined by vectors $\mathbf{R}_{i}+\Delta \mathbf{R}_{i}$ (in the inertial system) or by vectors $\mathbf{r}_{i}+\Delta \mathbf{r}_{i}$ (in the moving system). The absolute velocity is $\mathbf{V}_{i}+\Delta \mathbf{V}_{i}$. The angular momentum is equal to $\mathbf{K}_{O_{1} 1}(t+\Delta t)=\sum_{i}\left(\mathbf{r}_{i}+\Delta \mathbf{r}_{i}\right) \times\left(m_{i}-\Delta m_{i}\right)\left(\mathbf{V}_{i}+\Delta \mathbf{V}_{i}\right)$.

- mass $m=\sum_{i} \Delta m_{i}$ that leaves the missile body with absolute velocity $\mathbf{V}_{i}+\mathbf{U}_{\text {rel_i }}$. If the missile is driven by the engine with one nozzle outlet, this velocity is defined by formula (4.7). The angular momentum of this mass is equal to $\mathbf{K}_{O_{-} 2}(t+\Delta t)=$ $\sum_{i}\left(\mathbf{r}_{i}+\Delta \mathbf{r}_{i}\right) \times \Delta m_{i}\left(\mathbf{V}_{i}+\mathbf{U}_{\text {rel_ } i}\right)$. 
The change of the angular momentum of the system is equal to

$$
\begin{aligned}
& \Delta \mathbf{K}_{O}=\mathbf{K}_{O \_1}(t+\Delta t)+\mathbf{K}_{O_{-2}}(t+\Delta t)-\mathbf{K}_{O}(t)=\sum_{i} \mathbf{r}_{i} \times m_{i} \Delta \mathbf{V}_{i} \\
& \quad+\sum_{i} \Delta \mathbf{r}_{i} \times m_{i} \mathbf{V}_{i}+\sum_{i} \mathbf{r}_{i} \times \Delta m_{i} \mathbf{U}_{r e l_{\_} i}
\end{aligned}
$$

On the basis of (5.2), it is possible to calculate the derivative of the angular momentum $\mathbf{K}_{O}$ with respect to time

$$
\frac{d \mathbf{K}_{O}}{d t}=\sum_{i} \mathbf{r}_{i} \times m_{i} \frac{d \mathbf{V}_{i}}{d t}+\sum_{i} \mathbf{V}_{P_{i} / O} \times m_{i} \mathbf{V}_{i}-\sum_{i} \mathbf{r}_{i} \times \dot{m}_{i} \mathbf{U}_{r e l \_i}
$$

The same derivative can be calculated directly by differentiating expression (5.1)

$$
\frac{d \mathbf{K}_{O}}{d t}=\sum_{i} \mathbf{V}_{P_{i} / O} \times m_{i} \mathbf{V}_{i}+\sum_{i} \mathbf{r}_{i} \times \frac{d\left(m_{i} \mathbf{V}_{i}\right)}{d t}
$$

The second component in (5.4) is the moment of external forces acting on the missile relative to the pole $O$. This moment has the following components in the system $O x y z \mathbf{M}_{O}=[L, M, N]$.

By comparing expressions (5.3) and (5.4), we obtain

$$
\mathbf{M}_{O}=\sum_{i} \mathbf{r}_{i} \times m_{i} \frac{d \mathbf{V}_{i}}{d t}-\sum_{i} \mathbf{r}_{i} \times \dot{m}_{i} \mathbf{U}_{r e l \_} i
$$

If one takes into account expression (2.4) defining the absolute acceleration of any point of the missile, and assumes that the missile has one nozzle, equation (5.5) takes the final form

$$
\begin{aligned}
\mathbf{S} \times & \left(\frac{d^{\prime} \mathbf{V}_{0}}{d t}+\boldsymbol{\omega} \times \mathbf{V}_{O}\right)+\mathbf{I} \boldsymbol{\varepsilon}+\boldsymbol{\omega} \times(\mathbf{I} \boldsymbol{\omega})+\sum_{i} m_{i} \mathbf{r}_{i} \times \mathbf{a}_{r e l \_i} \\
& +2 \sum_{i} m_{i} \mathbf{r}_{i} \times\left(\boldsymbol{\omega} \times \mathbf{V}_{r e l_{\_} i}\right)=\mathbf{M}_{O}+\mathbf{M}_{T}
\end{aligned}
$$

where $\mathbf{M}_{T}$ is the moment of thrust $\mathbf{M}_{T}=\sum_{i} \mathbf{r}_{i} \times \dot{m}_{i} \mathbf{U}_{r e l \_}=\mathbf{r}_{E} \times \dot{m} \mathbf{U}_{r e l}$. This moment has the following components in the system Oxyz: $\mathbf{M}_{T}=\left[L_{T}, M_{T}, N_{T}\right] . \mathbf{I}$ is the matrix of inertia moments.

Equations (5.6) can be simplified by taking into account the assumption of axial symmetry of the missile. If the thrust lies on the axis of the missile, the final scalar form of this equation is as follows

$$
\begin{aligned}
& I_{x} \dot{P}=L \\
& I_{y} \dot{Q}+P R\left(I_{x}-I\right)-S_{x}(\dot{W}+P V-Q U)+2 Q \sum_{i} m_{i} x_{i} U_{r e l_{-} i}=M \\
& I_{z} \dot{R}+P Q\left(I-I_{x}\right)+S_{x}(\dot{V}+R U-P W)+2 R \sum_{i} m_{i} x_{i} U_{r e l_{\_} i}=N
\end{aligned}
$$

\section{The final form of the equations of motion}

Motion of the axisymmetric missile is described by equations (4.10) and (5.7) complemented with kinematic relations - we have the system of twelve differential equations

$$
\mathbf{A} \dot{x}=\mathbf{f}
$$

where $\mathbf{x}$ is the vector of flight parameters $\mathbf{x}=\left[U, V, W, P, Q, R, \Phi, \Theta, \Psi, x_{O}, y_{O}, z_{O}\right]^{\mathrm{T}}$. 
Non-zero elements of the matrix $\mathbf{A}$ are equal to

$$
\begin{array}{llll}
A_{11}=m & A_{22}=m & A_{26}=S_{x} & A_{33}=m \\
A_{35}=-S_{x} & A_{44}=I_{x} & A_{53}=-S_{x} & A_{55}=I_{y} \\
A_{62}=S_{x} & A_{66}=I_{y} & &
\end{array}
$$

whereas, the vector $\mathbf{f}$ is defined as follows

$$
\begin{aligned}
& f_{1}=F_{x}+T+m(R V-Q W)+S_{x}\left(Q^{2}+R^{2}\right)-m a_{x_{\_} r e l_{\_}}-\ddot{m}\left(x_{C}-x_{E}\right)-2 \dot{m} U_{\text {rel_C }} \\
& f_{2}=F_{y}+m(P W-R U)-S_{x} P Q-2 m R U_{\text {rel_C }}-2 \dot{m} R\left(x_{C}-x_{E}\right) \\
& f_{3}=F_{z}+m(Q U-P V)-S_{x} P R+2 m Q U_{\text {rel_C }}+2 \dot{m} Q\left(x_{C}-x_{E}\right) \\
& f_{4}=L \\
& f_{5}=M+P R\left(I_{y}-I_{x}\right)+S_{x}(P V-Q U)-2 Q \sum_{i} m_{i} x_{i} U_{\text {rel_l}} \\
& f_{6}=N+P Q\left(I_{y}-I_{x}\right)+S_{x}(P W-R U)-2 R \sum_{i} m_{i} x_{i} U_{r e l_{-} i} \\
& f_{7}=P+(Q \sin \Phi+R \cos \Phi) \tan \Theta \\
& f_{8}=Q \cos \Phi-R \sin \Phi \\
& f_{9}=\frac{Q \sin \Phi+R \cos \Phi}{\cos \Theta} \\
& f_{10}=U \cos \Psi \cos \Theta+V(\cos \Psi \sin \Theta \sin \Phi-\sin \Psi \cos \Phi)+W(\cos \Psi \sin \Theta \cos \Phi+\sin \Psi \sin \Phi) \\
& f_{11}=U \sin \Psi \cos \Theta+V(\sin \Psi \sin \Theta \sin \Phi+\cos \Psi \cos \Phi)+W(\sin \Psi \sin \Theta \cos \Phi-\cos \Psi \sin \Phi) \\
& f_{12}=-U \sin \Theta+V \cos \Theta \sin \Phi+W \cos \Theta \cos \Phi
\end{aligned}
$$

In equations (4.10), there are elements associated with relative motion of the mass center, while in equations (5.7) there are elements associated with motion of gas particles inside the body of the missile. They require determination of the relative velocity of mass center of the gas inside the missile. This will be described below.

\section{Determination of gas parameters inside the missile engine}

Due to the combustion process, mass $m$, static moments $S_{i}$ and inertia moments $I_{x}, I_{y}$ of the missile change. The quantity of fuel decreases in the combustion chamber and burning products leave the interior of the missile through the convergent-divergent nozzle (Fig. 3).

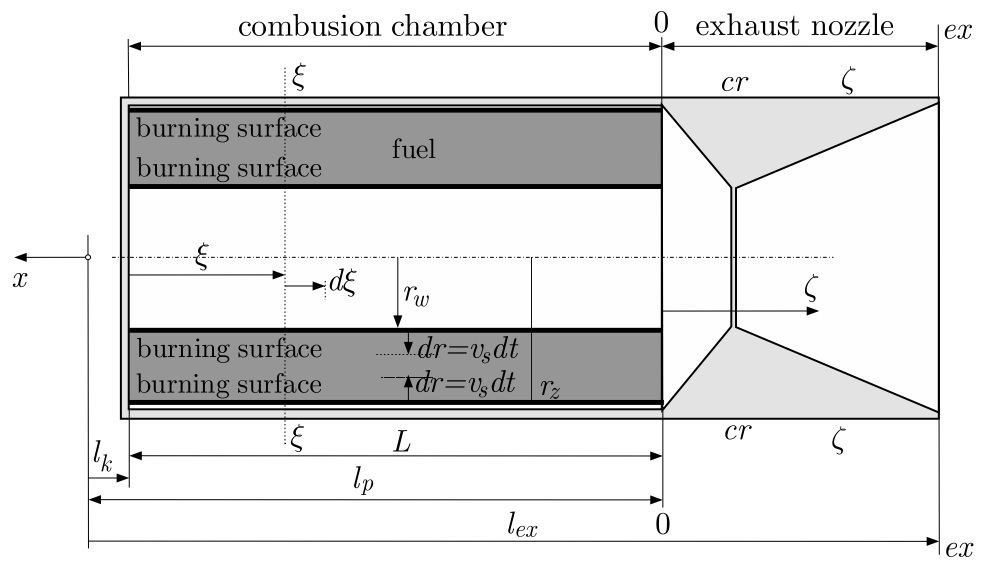

Fig. 3. Scheme of the solid fuel engine 
To determine the rule of change of fuel and these products, assumptions on the shape of the fuel and the space filled by gas should be made. Knowledge about physical parameters such as gas pressure $p$, temperature $T$, density $\rho$ and velocity $U$ is also necessary.

The following analysis will be carried out in which uniform velocity distribution of flow in each section of the missile engine is assumed (Mattingly, 2006; Torecki, 1984, Turner, 2009). This velocity remains parallel to the axis of the missile (the engine) $)^{2}$. In the calculations, the local coordinate systems associated with the combustion chamber $(\xi)$ and the nozzle $(\zeta)$ are introduced. This analysis also assumes that the mass flow rate is the same in each section of the nozzle. This means that the initial and final phases of the engine operation are omitted.

\subsection{The exhaust nozzle}

Energy of gases produced in the combustion chamber is converted into kinetic energy in the exhaust nozzle (Torecki, 1984). This nozzle is a supersonic de Laval nozzle, which means that the gas velocity increases in both the convergent as well as in the divergent parts. At the narrowest cross-section the gas velocity reaches the speed of sound. It is assumed that the flow in the nozzle is adiabatic and isentropic. As the gas velocity increases, and inside the nozzle is several times higher than the velocity at the inlet of the nozzle, it is assumed that the parameters at the inlet cross-section are stagnation parameters density $\rho_{0}$, temperature $T_{0}$, pressure $p_{0}$. Knowing the adiabatic index $k$, on the basis of the above assumptions, one can calculate velocity, density and temperature in any section of the nozzle inlet

$$
U=\sqrt{\frac{2 k}{k-1} \frac{p_{0}}{\rho_{0}}\left[1-\left(\frac{p}{p_{0}}\right)^{\frac{k-1}{k}}\right]} \quad \rho=\rho_{0}\left(\frac{p}{p_{0}}\right)^{\frac{1}{k}} \quad T=T_{0}\left(\frac{p}{p_{0}}\right)^{\frac{k-1}{k}}
$$

If the geometry $F(\zeta)$ of the nozzle is known, the pressure ratio $p / p_{0}$ can be found from the relation

$$
\frac{F}{F_{c r}}=\left(\frac{2 k}{k+1}\right)^{\frac{1}{k-1}} \sqrt{\frac{k-1}{k+1}} \frac{1}{\sqrt{\left(\frac{p}{p_{0}}\right)^{\frac{2}{k}}-\left(\frac{p}{p_{0}}\right)^{\frac{k+1}{k}}}}
$$

The mass flow rate in the nozzle can be described by the relation

$$
\dot{m}_{n o z z l e}=F_{c r}\left(\frac{2}{k+1}\right) \sqrt[\frac{1}{k-1}]{\frac{2 k}{k+1}} \sqrt{p_{0} \rho_{0}}=F_{c r} \frac{\left(\frac{2}{k+1}\right)^{\frac{1}{k-1}} \sqrt{\frac{2 k}{k+1}}}{\sqrt{R T_{0}}} p_{0}=C F_{c r} p_{0}
$$

where $C$ is the discharge coefficient depending on the fuel type and geometry of the nozzle, $R$ - gas constant of combustion products.

\subsection{The combustion chamber}

The analysis assumes that the fuel has shape of a hollow cylinder with length of $L$, see Fig. 3. The initial inner and outer radii are respectively equal to $r_{w 0}$ and $r_{z 0}$. It is assumed that the combustion takes place on the inner and outer surface of the cylinder with the burning speed $v_{\text {burn }}$. At the time $d t$, its inner radius increases and the outer radius decreases by a value

\footnotetext{
${ }^{2}$ This is relative velocity occurring in equations $(5.7)_{2,3}$ taken with the sign $(-)$. For clarity, the indexing is abandoned, i.e. instead of $-U_{\text {rel_ } i}$ we apply $U$.
} 
$d r=v_{\text {burn }} d t$. After the time $t$, radii $r_{w}$ and $r_{z}$, cross-sectional area $F$ of the space occupied by the products of combustion and the burning surface $S$ are equal to

$$
\begin{array}{ll}
r_{w}(t)=r_{w 0}+\int_{0}^{t} v_{b u r n} d \tau & r_{z}(t)=r_{z 0}-\int_{0}^{t} v_{b u r n} d \tau \\
F(t)=\pi\left[r_{w}^{2}+\left(r_{z 0}^{2}-r_{z}^{2}\right)\right] & S=2 \pi\left(r_{w 0}+r_{z 0}\right) L
\end{array}
$$

The latter formula shows that the combustion surface is constant. Whereas, the cross-sectional area $F(t)$ changes because of the change of $r_{w}$ and $r_{z}$.

It is assumed that the pressure $p_{0}$ and temperature $T_{0}$ is the same in the entire combustion chamber, and the burning speed is determined by a power series combustion law $v_{\text {burn }}=A p_{0}^{n}+B$ (Kurov and Dolzhaskii, 1961), where $A, B$ and $n$ depend inter alia on the chemical composition of the fuel and temperature of the initial charge. The mass flow rate of the gas into the combustion chamber is equal to $\dot{m}_{\text {chamber }}=\rho_{\text {fuel }} v_{\text {burn }} S$, where $\rho_{\text {fuel }}$ is the fuel density. In the initial and final stages of the fuel combustion process $\dot{m}_{\text {chamber }} \neq \dot{m}_{\text {nozzle }}$, which means that the flow is nonstationary and the pressure in the combustion chamber is changed. However, the fundamental phase of missile engine working is done with $\dot{m}_{\text {chamber }}=\dot{m}_{\text {nozzle }}$. This is a result of the so-called self-adjustment of the engine, which keeps a constant pressure in the combustion chamber.

Using the continuity equation for sections $0-0$ and $c r-c r$ and, further, applying the equation of the state of gas, the gas velocity at the end of the combustion chamber can be calculated as

$$
U_{0}(L, t)=\frac{C F_{c r} p_{0}}{\rho_{0} F(t)}=\frac{C F_{c r}}{F(t)} R T_{0}
$$

It is noted here that the velocity $U_{0}$ changes during the combustion process since the crosssectional area $F(t)$ increases due to changes in both the inner radius $r_{w}$ and outer radius $r_{z}$ according to formula (7.4).

In order to calculate the change of velocity along the combustion chamber, it is considered that the gas velocity increases with proximity to the outlet. In the section $d \xi$ over time $d t$ the burned fuel mass is equal to $d m=d S v_{\text {burn }} d t \rho_{f u e l}$ ( $d S$ is an elementary surface of combustion for the $d \xi$ section). An increase in the mass flow rate inside the combustion chamber is $d \dot{m}=v_{\text {burn }} \rho_{\text {fuel }} d S$. Simultaneously, taking into account the constant velocity distribution in the cross section of the chamber $\xi-\xi$, the increase is equal to $d \dot{m}=\rho_{0} F d U$. Comparing these expressions, one can determine the change of velocity $d U=\left[v_{\text {burn }} \rho_{f u e l} /\left(\rho_{0} F\right)\right] d S$. In order to calculate the velocity $U$, it is necessary to know the relationship for the sectional area $F$ and for the combustion surface $S$ as a function of the coordinate $\xi$. We have

$$
U(\xi, t)=\frac{v_{\text {burn }} \rho_{\text {fuel }}}{\rho_{0}} \frac{1}{F(t)} \int_{0}^{\xi} \frac{\partial S(x, t)}{\partial x} d x
$$

If the fuel has shape of a hollow cylinder, then on the basis of (7.4), one has $\partial S / \partial x=$ $2 \pi\left(r_{w 0}+r_{z 0}\right)$. Taking into account the expression for the cross-sectional area $F(t)$ and performing integration, one can calculate the gas velocity at $\xi$

$$
U(\xi, t)=2 v_{\text {burn }} \frac{\rho_{\text {fuel }}}{\rho_{0}} \frac{\left(r_{w 0}+r_{z 0}\right) L}{r_{w}^{2}+\left(r_{z 0}^{2}-r_{z}^{2}\right)} \frac{\xi}{L}=U_{0} \frac{\xi}{L}
$$

This formula takes into account boundary conditions: $U=0$ for $\xi=0$ and $U=U_{0}$ for $\xi=L$. 


\section{Changes of the missile mass parameters}

\subsection{The change of the speed of mass}

In the time interval $d t$, along the entire length of the load, the mass occupying the volume $S v_{\text {burn }} d t$ is combusted. Knowing density $\rho_{\text {fuel }}$, one can calculate this mass $d m=2 \pi\left(r_{w 0}+\right.$ $\left.r_{z 0}\right) L v_{b u r n} d t \rho_{f u e l}$. So, the rate of change of fuel mass as well as of missile mass is equal to $\dot{m}=\dot{m}_{f u e l}=2 \pi\left(r_{w 0}+r_{z 0}\right) L v_{\text {burn }} \rho_{\text {fuel }}$. As can be seen, this rate is constant, which means that the component of equation $(4.10)_{1}$ comprising the second derivative is equal to zero $\ddot{m}=\ddot{m}_{f u e l}=0$.

\subsection{Mass of the fuel and missile}

Knowing the rate $\dot{m}$, we can calculate the fuel and missile masses at any point of time. If at the beginning they are $m_{\text {fuel_o } 0}$ and $m_{0}$, respectively, we have

$$
\begin{aligned}
& m_{\text {fuel }}(t)=m_{\text {fuel_o } 0}-2 \pi\left(r_{w 0}+r_{z 0}\right) L \rho_{\text {fuel }} v_{\text {burn }} t \\
& m(t)=m_{0}-2 \pi\left(r_{w 0}+r_{z 0}\right) L \rho_{\text {fuel }} v_{\text {burn }} t
\end{aligned}
$$

\subsection{Motion of the center of mass of the missile}

Equations (4.10) require determination of changes in the position of the center of mass and the relative velocity $U_{r e l_{-} C}$ and its relative acceleration $a_{x_{-} r e l_{-} C}$. During operation of the engine, the fuel mass decreases, while the remaining mass is unchanged. At the same time, the center of the decreasing fuel mass is constant. It means that

$$
x_{C}(t)=\frac{x_{\text {fus }} m_{\text {fusel }}+x_{\text {fuel }} m_{\text {fuel }}(t)+x_{\text {chamber }} m_{\text {chamber }}+x_{\text {nozzle }} m_{\text {nozzle }}}{m}
$$

The first and second derivatives of the above expression give the relative velocity and acceleration

$$
U_{\text {rel_C }}=\dot{m}_{f u e l} \frac{x_{f u e l}-x_{C}}{m} \quad a_{x_{-} r e l \_}=-2 \frac{\dot{m}_{f u e l}}{m} U_{r e l \_C}
$$

\subsection{Static and inertia moments}

On the adopted assumptions concerning the shape and way of the burning process, the static moment and inertia moments can be calculated. For all parts of the missile - fuselage, fuel and gases filling the combustion chamber and the nozzle it is necessary to know the distribution of mass. Table 1 shows necessary formulas which allow one to calculate all static and inertia moments.

\section{Members of the equations of motion depending on the relative velocity and relative acceleration}

There are elements comprising the relative velocity $U_{\text {rel }}$ of the gases moving inside the body of the missile in equations $(6.3)_{2,3}$. They are determined below assuming an uniform distribution of the relative speed in all sections of the engine. Calculations are performed for the combustion chamber and nozzle. 
Table 1

\begin{tabular}{|c|c|c|}
\hline $\begin{array}{c}\text { Static and } \\
\text { inertia moments }\end{array}$ & $\begin{array}{l}\text { Elementary mass } \\
\text { for calculation }\end{array}$ & Formula \\
\hline $\begin{array}{l}\text { Total static } \\
\text { moment } S_{x}\end{array}$ & \multicolumn{2}{|c|}{$S_{x}=S_{x_{-} f u s e l}+S_{x_{-} f u e l}+S_{x_{-} \text {chamber }}+S_{x_{-} \text {nozzle }}$} \\
\hline$S_{x_{-} f u s e l}$ & $d m$ & $S_{x_{-} \text {fusel }}=\iiint_{\text {fuselage }} x d m$ \\
\hline$S_{x_{-} f u e l}$ & $d m=\rho_{f u e l} \pi\left(r_{z}^{2}-r_{w}^{2}\right) d x$ & $S_{x_{-} f u e l}=\frac{1}{2} \rho_{f u e l} \pi\left(r_{z}^{2}-r_{w}^{2}\right)\left(l_{k}^{2}-l_{p}^{2}\right)$ \\
\hline$S_{x_{\text {chamber }}}$ & $d m=\rho_{0} F d x$ & $S_{x_{-} \text {chamber }}=\frac{1}{2} \rho_{0} \pi\left[r_{w}^{2}+\left(r_{z 0}^{2}-r_{z}^{2}\right)\right]\left(l_{k}^{2}-l_{p}^{2}\right)$ \\
\hline$S_{x_{n o z z l e}}$ & $d m=\rho(x) F(x) d x$ & $S_{x_{-} n o z z l e}=\rho_{0} \int_{l_{e x}}^{l_{p}} F(x)\left(\frac{p}{p_{0}}\right)^{\frac{1}{k}} x d x$ \\
\hline $\begin{array}{l}\text { Total inertia } \\
\text { moment } I_{x}\end{array}$ & \multicolumn{2}{|c|}{$I_{x}=I_{x_{-} f u s e l}+I_{x_{-} \text {fuel }}$} \\
\hline$I_{x_{-} f u s e l}$ & $d m$ & $I_{x_{-} \text {fusel }}=\iiint_{\text {fuselage }} r^{2} d m$ \\
\hline$I_{x_{-} f u e l}$ & $d m=\rho_{\text {fuel }} 2 \pi r L d r$ & $I_{x_{-} f u e l}=\frac{1}{2} \pi \rho_{f u e l} L\left(r_{z}^{4}-r_{w}^{4}\right)$ \\
\hline $\begin{array}{c}\text { Total inertia } \\
\text { moment } I_{y}\end{array}$ & \multicolumn{2}{|c|}{$I_{y}=I_{y_{-} f u s e l}+I_{y_{-} f u e l}+I_{y_{-} \text {chamber }}+I_{y_{-} n o z z l e}$} \\
\hline$I_{y_{-} \text {fusel }}$ & $d m$ & $I_{y_{-} \text {fusel }}=\iiint_{\text {fuselage }} x^{2} d m$ \\
\hline$I_{y_{-} f u e l}$ & $d m=\rho_{p} \pi\left(r_{z}^{2}-r_{w}^{2}\right) d x$ & $I_{y_{-} \text {fuel }}=\frac{1}{3} \rho_{\text {fuel }} \pi\left(r_{z}^{2}-r_{w}^{2}\right)\left(l_{k}^{3}-l_{p}^{3}\right)$ \\
\hline$I_{y_{-} \text {chamber }}$ & $d m=\rho_{0} \pi\left[r_{w}^{2}+\left(r_{z 0}^{2}-r_{z}^{2}\right)\right] d x$ & $I_{y_{-} \text {chamber }}=\frac{1}{3} \rho_{0} \pi\left[r_{w}^{2}+\left(r_{z 0}^{2}-r_{z}^{2}\right)\right]\left(l_{k}^{3}-l_{p}^{3}\right)$ \\
\hline$I_{y_{-} n o z z l e}$ & $d m=\rho(x) F(x) d x$ & $I_{y_{-} n o z z l e}=\rho_{0} \int_{l_{e x}}^{l_{p}} F(x)\left(\frac{p}{p_{0}}\right)^{\frac{1}{k}} x^{2} d x$ \\
\hline
\end{tabular}

\section{The combusion chamber}

Elementary mass of the gas in the section $d x$ of the combustion chamber is equal to $d m=\rho_{0} \pi\left[r_{w}^{2}+\left(r_{z 0}^{2}-r_{z}^{2}\right)\right] d x$. Its velocity is given by (7.6). Taking into account this relationship, one can obtain

$$
\sum_{i} m_{i} x_{i} U_{r e l \_i}=\pi v_{s} \rho_{p}\left(r_{w 0}+r_{z 0}\right)\left(l_{k} l_{p}^{2}-\frac{1}{3} l_{k}^{3}-\frac{2}{3} l_{p}^{3}\right)
$$

\section{The exhaust nozzle}

The sought expressions for the nozzle can be found based on the knowledge of density distribution $\rho(x)$ and velocity distribution $U_{\text {rel }}(x)$ along the nozzle. We have

$$
\sum_{i} m_{i} x_{i} U_{r e l_{-} i}=\int_{-l_{e x}+l_{p}}^{0}\left(\zeta-l_{p}\right) \rho F U_{\text {rel }} d \zeta
$$

This integral can be efficiently calculated applying numerical methods and solving the issue of gas flow through the nozzle, as described in Section 7.1. 


\section{Determination of external forces acting on the missile}

The right-hand side of equations (6.1) includes components of the resultant external force $\mathbf{F}$ acting on the missile and the resultant moment of external forces $\mathbf{M}_{O}$ relative to the pole $O$. The external force $\mathbf{F}$ is the sum of the weight $\mathbf{Q}$ and the aerodynamic force $\mathbf{R}$. Whereas, the moment $\mathbf{M}_{O}$ relative to the pole $O$ is the sum of the moments generated by the weight $\mathbf{M}_{Q}=\mathbf{r}_{C} \times \mathbf{Q}$ and the aerodynamic moment $\mathbf{M}_{a e r}$

$$
\mathbf{F}=\mathbf{Q}+\mathbf{R} \quad \mathbf{M}_{O}=\mathbf{M}_{Q}+\mathbf{M}_{a e r}
$$

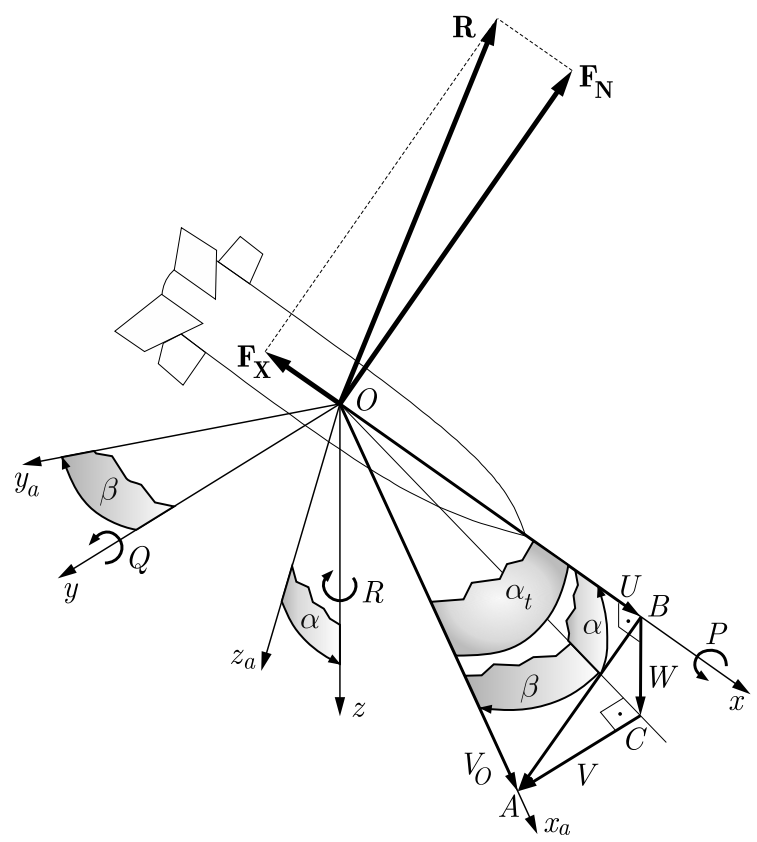

Fig. 4. Coordinate systems, aerodynamic forces, velocities and angles

The aerodynamic forces and moments can be divided into static and dynamic. The static forces and moments are determined on the basis of the value of the nutation angle $\alpha_{t}$ (Fig. 4). At the same time, the dynamic forces and moments are created when the missile is rotating. They have a damping character. The resultant aerodynamic force $\mathbf{R}$ is equal to the sum of forces: axial force $\mathbf{F}_{X}$ and normal force $\mathbf{F}_{N}, \mathbf{R}=\mathbf{F}_{X}+\mathbf{F}_{N}$ (Davis et al., 1958; McCoy, 2012; Rosser et al., 1947). The final expressions defining the components of the external force and moments are as follows

$$
\begin{aligned}
& F_{x}=-m g \sin \Theta-C_{x} \frac{\rho V_{\text {air }}^{2}}{2} S \\
& F_{y}=m g \cos \Theta \sin \Phi+\frac{\rho V_{\text {air }}^{2}}{2} S\left[C_{N \delta}\left(\frac{-V}{V_{\text {air }}}\right)+\left(\frac{P d}{V_{\text {air }}}\right) C_{N_{p} \delta}\left(\frac{W}{V_{\text {air }}}\right)+\left(\frac{R d}{V_{\text {air }}}\right)\left(C_{N q}+C_{N \dot{\alpha}}\right)\right] \\
& F_{z}=m g \cos \Theta \cos \Phi-\frac{\rho V_{\text {air }}^{2}}{2} S\left[C_{N \delta}\left(\frac{-W}{V_{\text {air }}}\right)+\left(\frac{P d}{V_{\text {air }}}\right) C_{N_{p} \delta}\left(\frac{-V}{V_{\text {air }}}\right)+\left(\frac{-Q d}{V_{\text {air }}}\right)\left(C_{N q}+C_{N \dot{\alpha}}\right)\right] \\
& L=\frac{\rho V_{\text {aer }}^{2}}{2} S d C_{l p}\left(\frac{P d}{V_{\text {aer }}}\right) \\
& M=-x_{C} m g \cos \Theta \cos \Phi+\frac{\rho V_{\text {air }}^{2}}{2} S d\left[C_{M \delta}\left(\frac{W}{V_{\text {air }}}\right)+\left(C_{M q}+C_{M \dot{\alpha}}\right)\left(\frac{Q d}{V_{\text {air }}}\right)\right. \\
&\left.+\left(\frac{P d}{V_{\text {air }}}\right) C_{M_{p \delta}}\left(\frac{V}{V_{\text {air }}}\right)\right]
\end{aligned}
$$




$$
N=x_{C} m g \cos \Theta \sin \Phi+\frac{\rho V_{a i r}^{2}}{2} S d\left[C_{M \delta}\left(\frac{-V}{V_{a i r}}\right)+\left(C_{M q}+C_{M \dot{\alpha}}\right)\left(\frac{R d}{V_{\text {air }}}\right)+\left(\frac{P d}{V}\right) C_{M_{p \delta}}\left(\frac{W}{V_{\text {air }}}\right)\right]
$$

$V_{\text {air }}$ is the velocity at which the missile moves relative to the air $\mathbf{V}_{\text {air }}=\mathbf{V}_{O}-\mathbf{V}_{\text {wind }}$. All coefficients in brackets [...] determine the aerodynamic forces and moments. They depend on the nutation angle and the Mach number.

\section{Numerical example and conclusions}

Formulas $(6.2)_{1}-(6.2)_{5}$ show that during the combustion process there are additional forces and moments which depend on the mass flow rate, relative speed of the combustion products and their mass. In particular, there are additional moments depending on the angular velocities: $-2 Q \sum_{i} m_{i} x_{i} U_{\text {rel_ }} i$ and $-2 R \sum_{i} m_{i} x_{i} U_{\text {rel_ } i}$. They have a damping character as well as the aerodynamic damping moments (members containing $\left(C_{M q}+C_{M \dot{\alpha}}\right)$ in expressions $(10.2)_{5}$ and $\left.(10.2)_{6}\right)$. To compare both damping moments, the following derivatives are analyzed

$$
M_{\text {aer }}^{Q}=\left(C_{M q}+C_{M \dot{\alpha}}\right) \frac{\rho V_{\text {air }}}{2} S d^{2} \quad M_{j e t}^{Q}=-2 \sum_{i} m_{i} x_{i} U_{\text {rel_ } i}
$$

To do it, numerical simulation of flight for a homing guided missile has been performed. For this reason, additionally control laws are also determined on the basis of methods described in (Krasovskii, 1969, 1973; Locke, 1955; Neupokoev, 1991; Shneyder, 1998; Siouris, 2004). A lot of various variants of the initial conditions both for the missile and for the maneuvering target are used. Below, exemplary results for one variant of target flight are presented - a turn maneuver with increasing altitude. It is assumed that the target is detected by the missile at the distance of $30 \mathrm{~km}$ and initial altitude of $5 \mathrm{~km}$. Its velocity is $100 \mathrm{~m} / \mathrm{s}$. The initial conditions for the missile (class Patriot MIM-104) are as follows: pitch angle $45^{\circ}$, yaw angle $0^{\circ}$. The working time of the engine is $12 \mathrm{~s}$. The initial mass of the missile is $910 \mathrm{~kg}$, mass of the fuel is $508 \mathrm{~kg}$. The control system starts to work two seconds after blast-off.
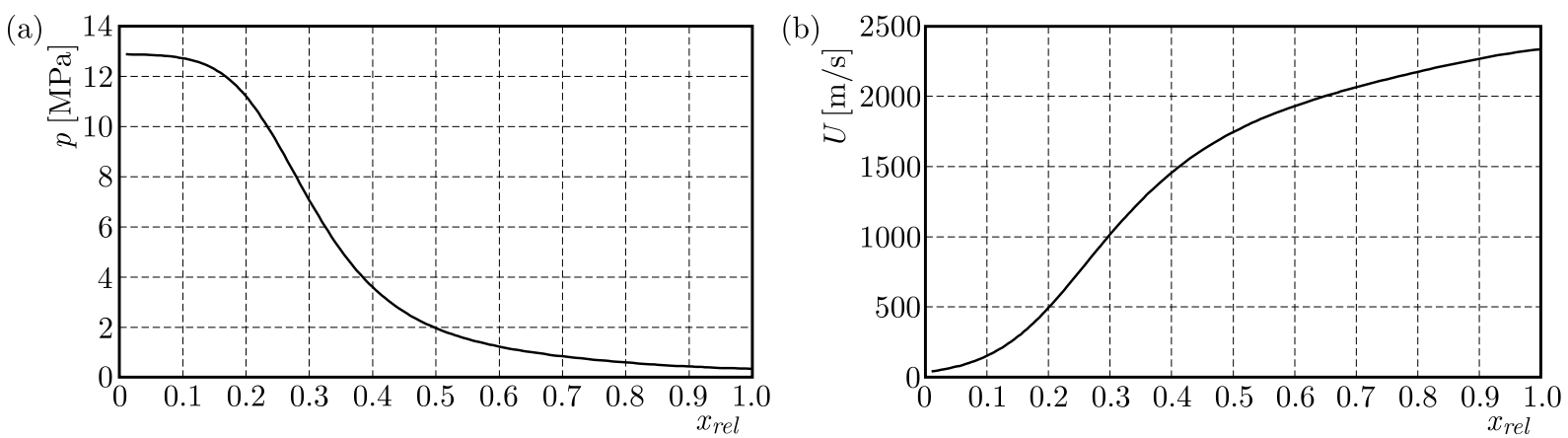

Fig. 5. Gas pressure (a) and gas velocity (b) along exhaust nozzle

It is assumed that the combustion temperature for nitrocellulose solid propellant grain is $T_{0}=2500 \mathrm{~K}$. The calculations show that for this temperature the pressure in the combustion chamber is $p_{0}=12.9 \mathrm{MPa}$, the burning speed $v_{\text {burn }}=8 \mathrm{~mm} / \mathrm{s}$ and the relative velocity at the nozzle exit is $U r_{r e l}=2336 \mathrm{~m} / \mathrm{s}$. These parameters give a thrust equal to $T=107.08 \mathrm{kN}$. Exemplary changes of gas parameters in the exhaust nozzle are shown in Figs. 5a and 5b. The defined by (11.1) derivatives are presented in Fig. 6a. We can see that the derivative $M_{\text {jet }}^{Q}$ is constant during the combustion process. The absolute value of the derivative $M_{\text {aer }}^{Q}$ depends nonlinearly on $V_{\text {air }}$ because the coefficients $\left(C_{M q}+C_{M \dot{\alpha}}\right)$ depend on the Mach number (Fig. 6b). Figure 6a proves that the aerodynamic derivative $M_{a e r}^{Q}$ is many times greater than $M_{j e t}^{Q}$. Therefore, any 

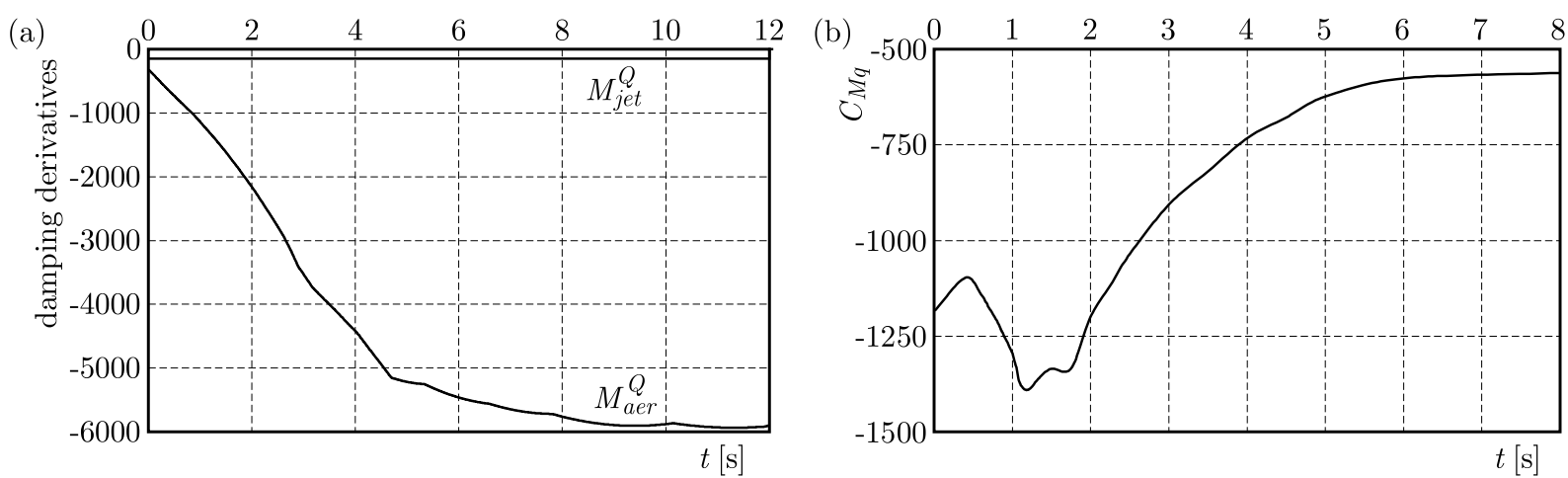

Fig. 6. (a) Damping derivatives; (b) coefficient $C_{M q}$

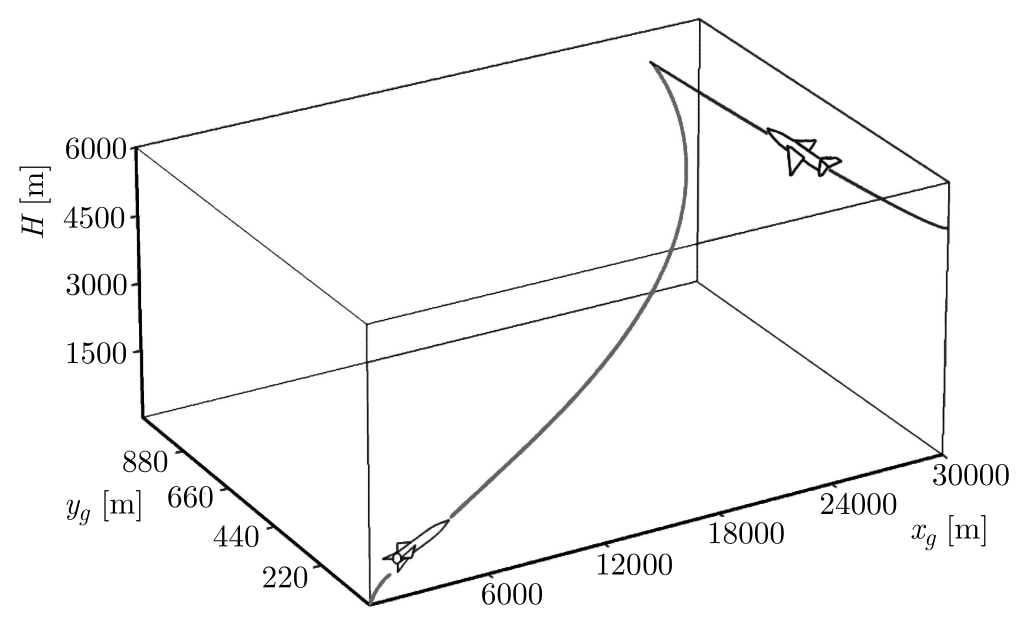

Fig. 7. Missile and target trajectories
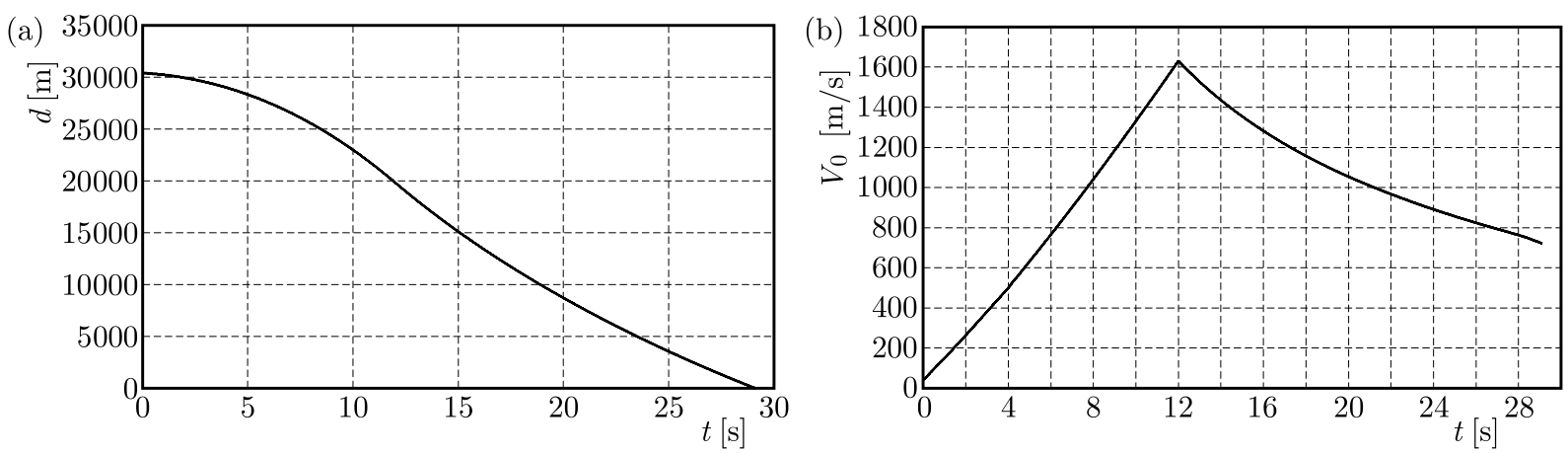

Fig. 8. (a) Missile-target distance; (b) missile velocity

(a)

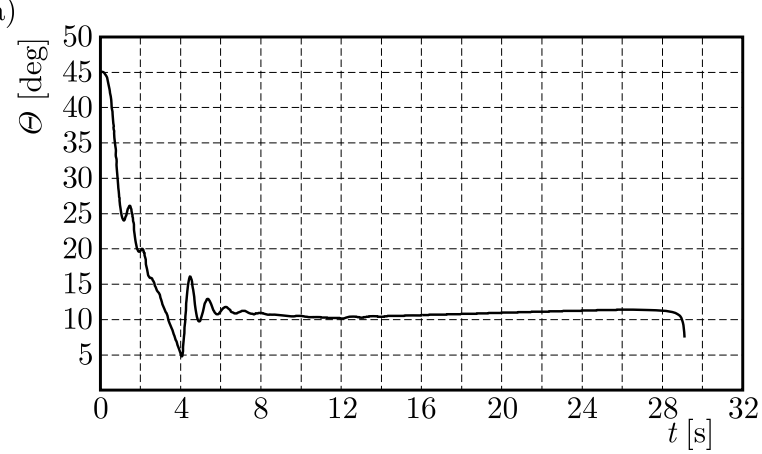

(b)

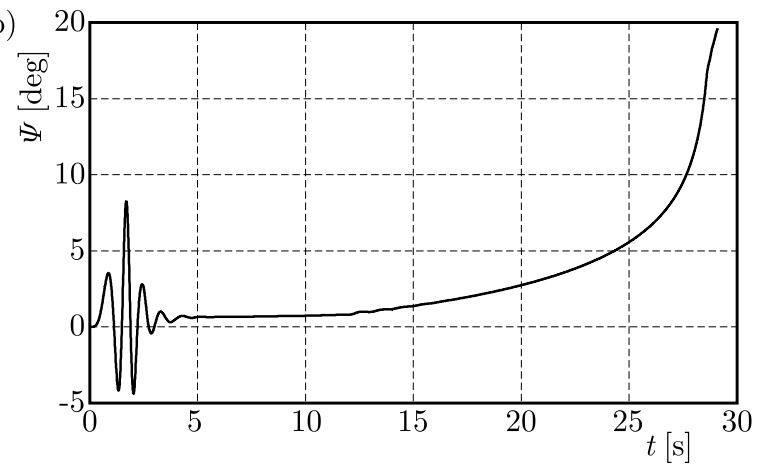

Fig. 9. (a) Pitch angle; (b) yaw angle 
important influence of the jet damping effect on the missile atmospheric flight has not been found. The trajectories of the missile and the target are shown in Fig. 7. We can see that the maneuvering target is hit - a distance between the missile and the target decreases to zero, see Fig. 8a. Figure 8b presents velocity of the missile which grows during engine operation and next decreases. The pitch and yaw angles are presented in Figs. 9a and 9b.

Other obtained results also show that the jet damping effect can be neglected for maneuvering missiles when angular velocities are not equal to zero. The aerodynamic forces and moments are still of minor importance.

\section{References}

1. Davis L., Follin J.W., Blitzer L., 1958, Exterior Ballistics of Rockets, D. Van Nostrad Company Inc.

2. Dimitrevski A.A., 1972, Exterior Ballistics (in Russian), Publishing House "Maschinostroenie"

3. Krasovsky A.A., 1969, Analytical Design of Aircraft Control Loops (in Russian), Publishing House "Maschinostroenie"

4. Krasovsky A.A., 1973, Systems of the Automatic Flight Control and its Analytical Design (in Russian), Publishing House "Nauka"

5. Kurov V.D., Dolzhansky J.M., 1961, Fundamentals of Solid Propellant Rockets (in Russian), State Scientific and Technical Publishing "Oborongiz"

6. Locke A.S., 1955, Guidance, D. Van Nostrad Company Inc.

7. Mattingly J.D., 2006, Elements of Propulsion: Gas Turbines and Rockets, AIAA Education Series

8. McCoy R.L., 2012, Modern Exterior Ballistics, Schifler Publishing Ltd.

9. Neypokoev F.K., 1991, Firing of anti-aircraft missiles (in Russian), Voennoe Izdatelstvo

10. Quarelli M.B., Cameron J., Balaram B., Baranwal M., Bruno A., 2014, Modeling and simulation of flight dynamics of variable mass system, AIAA Space Conference and Exposition, San Diego, 2014-4454

11. Rosser J., Newton R., Gross G., 1947, Mathematical Theory of Rocket Flight, McGraw Hill Book Company Inc.

12. Thomson W.T., 1965a, Equations of motion for the variable mass system, AIAA Journal, 4, 4, $766-768$

13. Thomson W.T., 1965b, Jet damping of solid rocket: theory and flight results, AIAA Journal, $\mathbf{3}$, $3,413-417$

14. Thomson W.T., 1986, Introduction to Space Dynamics, Dover Publications Inc.

15. Torecki S., 1984, Rocket engines (in Polish), Wydawnictwa Komunikacji i Łączności

16. Turner M., 2009, Rocket and Spacecraft Propulsion - Principles, Practice and New Developments, Springer Verlag

17. Shneydor N.A., 1998, Missile Guidance and Pursuit: Kinematics, Dynamics and Control, Horwood Publishing

18. Siouris G.M., 2004, Missile Guidance and Control Systems, Springer Verlag 